Marija Maletić ${ }^{1}$, Dušan Barać ${ }^{2}$, Vuk Rakočević ${ }^{3}$, Tamara Naumović ${ }^{2}$ Artur Bjelica $^{5}$ ${ }^{1}$ DXC.Technology - Enterprise Services, Belgrade, Serbia ${ }^{2}$ University of Belgrade, Faculty of Organizational Sciences, Serbia

${ }^{3}$ Embassy of Montenegro in the Republic of Serbia

${ }^{5}$ University of Novi Sad, Faculty of Medicine, Serbia

\title{
Scaffolding E-voting in Developing Countries
}

DOI: 10.7595/management.fon.2019.0006

\begin{abstract}
:
Research question: This paper discusses e-voting as an integral part of e-government in developing countries. E-voting enables automation of casting and counting votes. Motivation: The main goal was to investigate the readiness of both the public sector and citizens for switching from common "paper" voting method to electronic system-based voting. In order to improve democracy and trust in the election process (IDEA, 2011), the e-voting system should be reliable, accurate and secure (Mauw, Verschuren, \& de Vink, 2007). The example that should be followed is the Estonian e-voting system (Drechsler, 2004). Idea: We proposed a comprehensive e-voting model that includes five components: e-voting services, IT infrastructure, participants' registration, components integration and a system for counting and reporting. Security is the most important issue that should be overcome by using the cryptographic protocols. Reliability and availability of the evoting system should not be neglected, and it should be able to save all verified casted votes. Data: In order to examine the readiness and awareness of e-voting potentials in a developing country, we conducted a survey that included 152 persons from three different groups: individuals, legal entities and e-government employees. Tools: In order to establish relationships among opinions of the three groups of respondents to the survey that was conducted, a comparative analysis was performed. For this purpose the questions were divided into four categories. Findings: As it was expected, the study results have showed that survey participants find security, lack of qualified staff and mistrust of the older generations towards new technologies as the crucial issues in developing and implementing the e-voting model. Based on the responses the cost reduction, ease of use and efficiency improvement are recognized as categories that could be realized by implementation of the e-voting system. Contribution: The results show the respondents' opinion regarding the e-voting system introduction as a legitimate voting tool. It can reduce abuse, voting costs and manual errors.
\end{abstract}

Key words: E-voting, e-government services, m-voting, e-voting applications

JEL Classification: M14, M15, O32, O33, O35

\section{Introduction}

The omnipresence of the Internet and its usage in numerous life spheres contribute to the transparency and information availability. This way the Internet becomes the starting point of a lot of research. Even the public opinion research by using the Internet technologies is raised onto a higher level and the possibilities of the online world are increasingly used (Maletic, Barac, Naumovic, Bogdanovic, \& Radenkovic, 2018).

E-government has an aim to improve quality, accuracy, management and service efficiency (Lee, 2010), (Spacek, 2014). E-government services serve citizens, companies and other government organizations (Veljkovic, Bogdanovic-Dinic, \& Stoimenov, 2011). This model increases transparency, information and services availability (Markovic, Stoimenov, Vojinovic, \& Milentijevic, 2008). Process automation leads to a reduction of the human factor, and thus a better service. As one of the most complex services, electronic voting resolves the issues of accuracy, reliability, security, but also automation the process of counting votes, which decreases the risk of abuse. Electronic voting refers to the use of electronic systems for voting and counting votes (IRMA, 2015). A system for electronic voting implies complex infrastructure that should meet the criteria of scalability, availability, security, reliability and profitability. An important aspect of electronic voting process is security in order to preserve vote authenticity but also to prevent abuse of submitted votes. 
The social dimension of further development and application of the e-government system and therefore electronic voting is of great importance for raising public awareness about possibilities of new technologies. E-society has an aim to increase labour efficiency, economic growth, increasing employment rate and raising the overall life quality of all citizens (Reddick \& Frank, 2007). The mechanism of information society represents support for development of e-business. Applying the concept of electronic business implies major changes of internal operating procedures of administration, as well as adjusting the new operating modes, including stable partnerships with the private sector. In addition to cost reduction and economic growth, electronic government aims to increase services availability, improves operations, and affects the formation of e-society and increase in transparency and accessibility (Anthopoulos, Siozos, \& Tsoukalas, 2007).

E-government services meet some of the main preconditions for sustainable business such as providing services in a more efficient way and with more quality than traditional principles, taking into consideration the environment state and needs of the wider entirety before the use itself (Anderson, 2006; Rennie, 2008). An especially important aspect of e-government services and e-voting is social responsible business by putting into focus the end users' needs. New technologies allow public sector's services to be more transparent, so that the current, traditional relationship between public administration bodies and its users is raised to a whole new level, where the services are adapted to the users (Internet access, meeting appointments, online documents ordering, etc.), care is taken for entire society and community (large savings in communication, transport, working material, etc.), awareness of the importance of new technologies is spread and the education of society is performed (Sandoval-Almazan \& Gil-Garcia, 2012). This way, e-democracy is promoted in a more effective way.

The subject of this paper is examination of the possibility to apply modern information technologies, the Internet and the concept of electronic government for the implementation of the voting process for the purposes of e-government of the Republic of Serbia. Currently, the voting process in Serbia is still conducted in the traditional manner which implies organizing voting stations with ballot boxes and manual vote counting. One of the main motives for using electronic voting systems is vote counting. The voting concept does not necessarily refer only to the parliamentary, presidential or local elections, but also to the elections of different organizations, schools or faculties.

The aim of this paper is to determine to what extent it is possible to introduce e-voting into the voting system of the Republic of Serbia. For this purpose a survey of three groups of respondents was conducted. The goals which should be achieved by implementation the e-voting model are increasing the efficiency of the e-voting process, improvement of cooperation of the state and the population, increasing the velocity of vote counting and reducing costs of the voting process organization, minimization of the human factor and adapting the voting process to the citizens with special needs. The main hypothesis tested in this paper is $(\mathrm{H} 1)$ :

There is a huge potential and high level of readiness among different stakeholders for introducing e-voting as an e-government service.

\section{E-voting as an e-Government Service}

E-government refers to the use of information and communication technologies for exchanging information, providing services and operations of national authorities and institutions with individuals and corporate entities, as well as among the branches of the government itself (Brown, 2003), (OECD, 2003). It may be said that electronic government represents online information exchange and thus provides services to the corporate entities and other organizations or agencies (Vrhovsek \& Spalevic, 2011), (Soliman \& Affisco, 2006). Digital governance includes both delivery of public services and citizen participation in governance (Nica, Popescu, Nicolaescu, \& Constantin, 2014). E-government appraisals should explain how people use the Internet not only for their private tasks but also as a means of involving them in public affairs (Sakowicz, 2004). In addition to facilitating the activities of competent authorities, e-services provide a better outcome; reduce costs, increase the speed and accuracy of transactions management. This has a favorable influence on the economy since it implies an efficient access to the government services and thus reduces costs of administrative functions (Al-Hakim, 2007). This manner increases transparency and information accessibility, as well as services availability (Kreca \& Vaskovic, 2013). One of the most important aspects of e-government development is the overall society development, process automation and greater efficiency.

G2C includes the information dissemination to the public as well as providing basic services to the citizens. As one of the most important G2C service of e-government, e-voting is a service which is intended for registered voters, e.g., persons entitled to vote - adults (Park, 2007). E-voting refers to the voting process 
using electronic systems, including punched cards, optical systems for vote scanning, as well as special voting kiosks. E-voting, in addition to voting via the Internet, may also involve voting via fax, telephone or private computer networks. Systems for e-voting are also considered to be the vote counting systems, with special attention paid to the proper functioning of the system in terms of compliance with defined rules. The main advantage of using the electronic voting system is the remote access which ensures accessibility to the persons with special needs. E-voting is often seen as a tool for advancing democracy, building trust in electoral management, adding credibility to election results and increasing the overall efficiency of the electoral process (IDEA, 2011).

Many countries successfully implemented the e-voting process. The Estonian case is the first case worldwide of a country that has actually passed overall e-voting laws (Drechsler, 2004). There are numerous applications developed for this purpose. The three leading universities in Lithuania developed the application Manobalsas for the purposes of Lithuanian parliamentary elections in 2008 (Ramonaite, 2010). However, there is a security issue since it is very important to adequately confirm voter's personal data, but without a possibility to trace the vote, in order to preserve voting secrecy. Therefore, it is important for the e-voting system to be reliable, to store voter's personal data and each vote, and to adequately count all the votes.

It is important to mention that e-voting systems are not perfect; in fact they have many flaws (Alkassar \& Volkamer, 2007). There may be software bugs, security holes, so the reliability of such systems is questionable. If the e-voting system is not reliable, it is possible that a certain amount of votes may be lost, or the voter to be marked wrongly, some votes incorrectly stored, etc. Problems also may be physical in a manner of insufficient capacity of the storage medium which stores the data and votes. A particular problem can be training the election commission, which implies a completely different approach and training requires the provision of the best support to the voters in order to use voting machines correctly. However, some research stresses that the main problem for electronic improvement could be political factors and institutional capacities, since the lack of these resources may influence the stagnation of e-government development (Frias-Aceituno, Garcia-Sanchez, \& Rodriguez-Dominguez, 2014).

Features of a good e-voting system (Mauw, Verschuren, \& de Vink, 2007) are as follows:

- Accuracy - not to allow vote changing; invalid vote shouldn't be considered;

- Reliability - only registered voters can vote; voters can vote only once; it cannot lose any data;

- Verification - vote counting should be done correctly; each vote must be properly recorded;

- Security - voting must be completely anonymous.

Will the concept of e-voting compensate for the lack of voters' interest in voting, when it comes to the modern voting methods? The number of those who do not vote will probably be reduced. However, traditional voting is not the main issue why some citizens do not participate in voting. If voters do not feel that they can make a difference with their votes, it is likely that they will still be disinterested in elections participation (Ke \& Wei, 2004).

To improve the voting process, it is considered that implementation of the system which sends an electronic invitation for voting can contribute to the improvement of this process, as well as electronic voters' authentication, providing more information about the candidates and their programmess and electronic publication of results.

These suggestions may reduce voting costs. Therefore, introducing such a voting system on the territory of the Republic of Serbia would mean more precision and accuracy during the vote counting and getting objective voting results.

The example of the e-voting system is the Direct Recording Electronic Voting System - DRE. These devices have a touch screen where a voter can see ballot and on his/her clicking the desired choice, the terminal keeps the vote and stores it on the memory card, disc or other memory medium (Hite, 2004).

In addition to the e-voting systems which facilitate the voting act, voters should get guidelines for the voting. These guidelines can be implemented into the voting advice system, which can provide a better insight into a candidate's political preferences, and thus it could help the voter to choose the candidate more easily (Fossen \& Anderson, 2014). As a support to the system for e-voting, voting advice applications (VAAs) provide the voter with a possibility to be informed about detailed political preferences of the candidates. This way the voter gets more information and a possibility to make a decision on the selection of candidate.

Voting Advice Applications - VAAs are interactive tools based on the Internet approach and provide information about candidates during elections (Ladner \& Pianzola, 2014). The use of voting advice 
applications is considered to have a positive impact on voting turnout (Ladner \& Pianzola, 2010), because this way the voters obtain more information about candidates, which can help them choose the one whose preferences match with their own, thus the interest of voters for participating in the voting process can be increased. The Vote Match Europe is an example of the VAA, hence it was developed as a quiz, hence based on the respondents' answers to the questions, it gets as a result the party whose political appearance is the most likely to match their own political preference. Statistics has found that respondents were more aware of political differences after they solved the quiz, but also the quiz helped them to get more information than they had, and some of the respondents changed their minds as to for whom to vote (Vote Match Europe, 2014), (Vote Match UK, 2014). Research has shown that the use of VAAs can be useful in non-ideological populist parties that can adapt the preferences of an average voter (Ramonaite, 2010).

It is important to emphasize that e-voting does not refer only to the governmental elections, but it is widely used for municipal elections, as well as for regulation adoption purposes and other political oriented elections. On the other hand, the e-voting system can be used for nominations, awards, contests and other social voting events. Online polls can also be considered as a form of e-voting system. Numerous online voting tools are developed for the purpose of measuring the public opinion and they can be designed in accordance to the examiner's research tendencies. Some of free online voting tools are: BigPulse (), EasyPolls (), Poll-maker (), etc.

E-voting can be associated with crowd voting. Crowd voting, as a subset of the crowdsourcing process, refers to the voting process where a crowd can vote on a certain topic by means of Internet communities. The full implementation and importance of crowd voting will be recognized in the future. The example of crowd voting is the web site Tricider (), where users can enter their questions and other users can provide their ideas regarding the question. This is also a good example of implementation of the the brainstorming Internet model. One more example of crowd voting developed to gain community opinions was voting for the American Idol show. Audience can vote via application or directly online - via a web site or via their Facebook account.

The conclusion is that the term "e-voting" can be related not only to governmental elections, but to social online voting, for example for the best destination, song, movie, etc.

\section{E-voting Process and Proposed Model}

In order to determine the voters' identification it is necessary to develop a module which will check whether the voter is registered. Depending on e-voting technology (whether it implies access from a computer or mobile phone), we suggest the following steps in the e-voting process:

1. Voter starts with the verification process:

a. If the voting is performed via a PC, the prerequisite is using the ID card and appropriate card reader. Voter inserts the ID card and opens a portal for e-voting.

b. If voting is performed via a mobile phone, the voter should open the e-voting application where he can enter the mobile number in order to receive the SMS with control code (PIN1)

2. Voter's verification:

a. In case of PC voting, the voter should enter his PIN1 of corresponding ID card

b. In case of mobile voting, the voter should enter his PIN1 obtained via SMS

3. Confirmation of the voter's identification - after comparing the entered PIN and the list of registered voters, the system sends to the voter a confirmation that the voting process can start

4. The selection of desired candidate - the system shows to the voter a list of registered candidates

5. Voter selects the desired candidate

6. System encrypts the vote and stores it on a server

7. Voter confirms his choice by digital signature, i.e., by entering the control code (PIN2)

8. System sends to the voter a confirmation of a successfully stored vote.

In order to prevent intrusions into the e-voting system the cryptographic protocols are used. The entire communication process is covered by cryptographic protocols, starting with voter identification, through vote recording to vote counting while maintaining the voter's anonymity. The use of these protocols leads to the keeping vote secrecy, as well as achieving the transparency of a system so that all participants have confidence in such a system, which leads to a successful voting process as a very important aspect of Internet voting. 


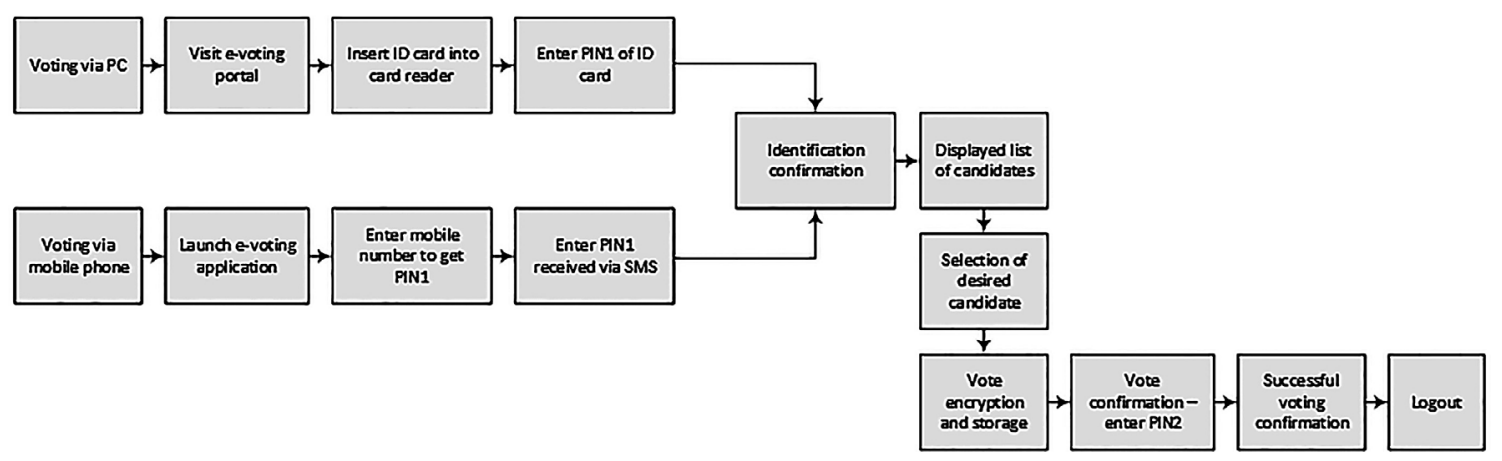

Figure 1: E-voting workflow

E-voting systems can be a target for unwanted intrusions. Attacks on these systems can be categorized into: program code changing, interface simulation, attacks on ID labels, vote changing, malicious applications on user's computer, attacks on servers, identity theft (Malidzan, 2011). The issues that are specific for the e-voting systems, especially when it comes to Internet voting, can be related to: voter's authentication, ballot privacy, ballot integrity, reliable vote transfer to the server, prevention of multiple voting, protection against attacks from client's side, protection against service attacks refusal on servers.

It is important to note that voting via personal computer is quite an unreliable way of voting, since it is an uncontrolled environment. Given that each protocol implemented in the process of Internet voting keeps the encrypted vote from unwanted intrusions and their changes without detection, there is a conclusion that the riskiest step is on the client's side, before the vote encryption.

Current laws and regulations control the irregularities related to the existing, traditional voting system so development is necessary in that area in order to control the possible malicious actions during e-voting (PIN stealing, Internet attacks, viruses, etc.) according to the regulations. The most important requirement that must be complied by the e-voting system is a complete vote secrecy from the moment of selecting the candidate until storing the vote to the server. The second requirement is that the vote which is transmitted from the voter to the server remains unchanged, i.e., that there is no possibility to change the vote by third parties. The server overload should also be taken into consideration since it can jeopardize voting results.

\subsection{The Structure of the Proposed Model}

The developed e-voting model should allow voter's authentication, recording and counting the votes, and reporting about voting results. For the purposes of developing such a system it is necessary to integrate the e-voting system, the IT infrastructure and the register of participants in the e-voting process.

Proposed e-voting model includes the following components:

- C1: E-voting services;

- C2: IT infrastructure;

- C3: Participants' register;

- C4: Components integration;

- C5: System for counting and reporting.

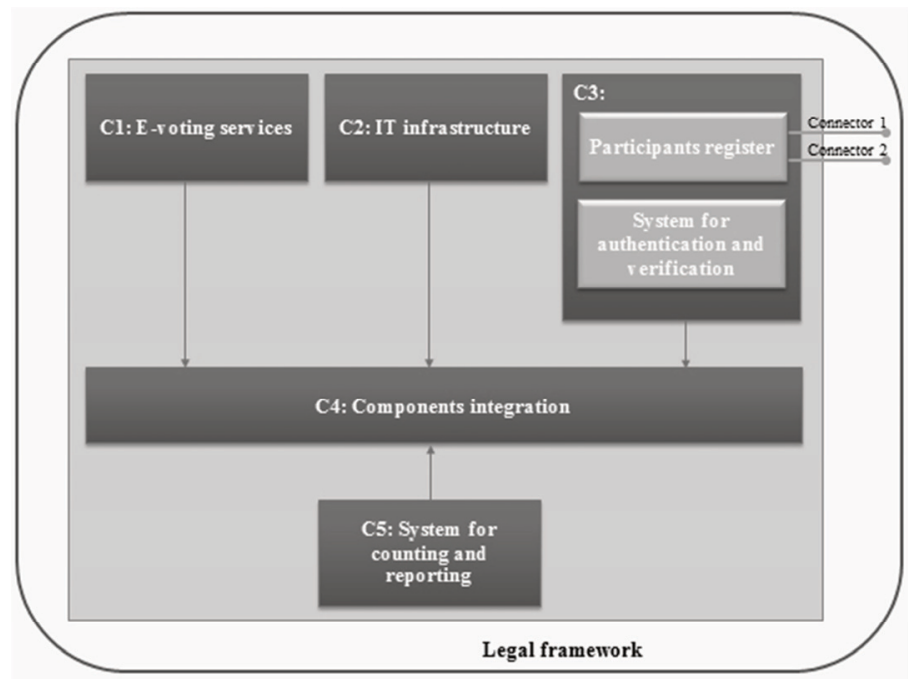

Figure 2: Structure of proposed e-voting model 
E-voting services can be realized through mobile and web services. These services communicate with the hardware, which refers to the e-voting devices and devices for authentication and verification.

The IT infrastructure of an e-voting system implies the set of hardware and software components. The hardware used for this purpose implies to the servers, network, card readers used for authentication and verification. Legal regulations are a crucial factor in voting in general, so the implemented application must meet the laws and regulations on electronic voting that apply to the territory of the Republic of Serbia.

In the voting process participants register contains information of registered voters and registered candidates. Information on registered voters is filled from an external source via connectors. The external source is the electronic government, and registered voters are all adult citizens of the Republic of Serbia. In order to determine the voter's identification, the system for authentication and verification provides security checks. The voter must have the ID card which is read by card reader and then he enters the PIN code of a card. After data comparison from the card and the entered PIN, the system sends a feedback to the voter that authentication process is successfully completed and that he can start voting. Names of registered candidates are entered manually by a chosen committee. The voter register and the candidate register are physically separated modules but they are integrated into participant register.

A partial e-voting system is obtained by integrating the e-voting services, IT infrastructure and participant register. The final product should be a web portal and a mobile application. Both voting methods can be adapted to persons with special needs. The integration of infrastructure components is shown in Figure 3.

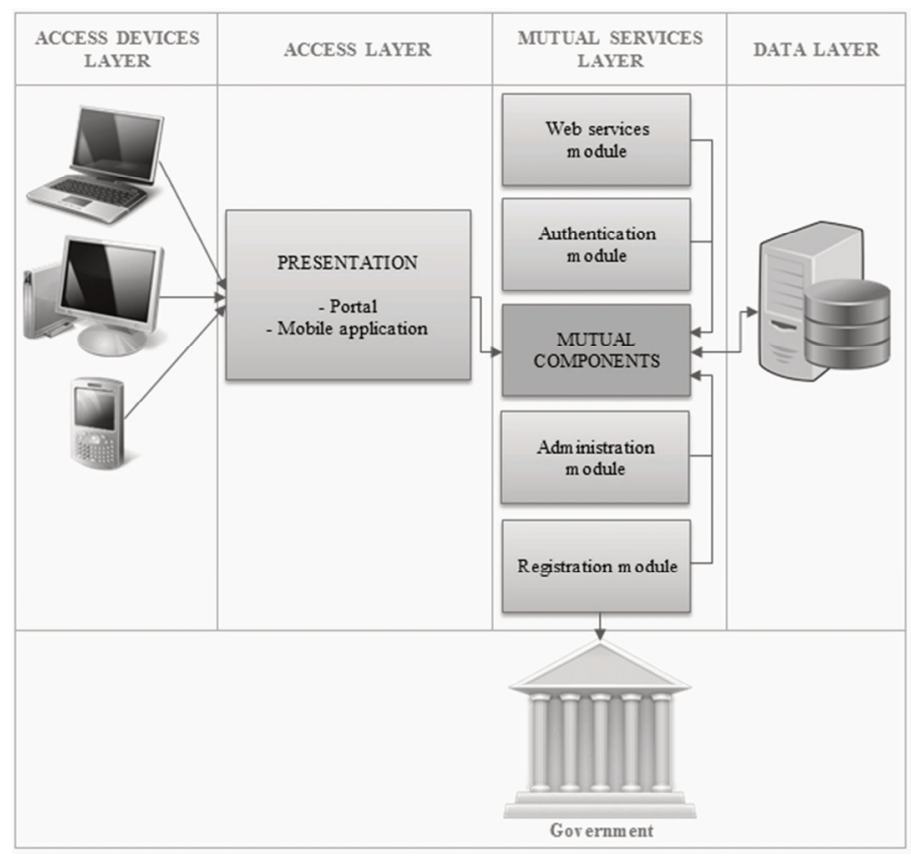

Figure 3: Graphical view of portal integration components

The result of the voter's action by using the e-voting system is a vote packed into ballot. The system for counting and reporting should decrypt the encrypted votes without revealing the personal information about the voter and then it should perform counting of valid votes. This system also provides reporting about voting results.

Each client (voter) connects to the e-voting portal via Internet connection from his personal computer, or accesses via a mobile application. On his logging on a portal, the system checks his personal data and compares them to the list of registered voters. The voter can be informed regarding candidates and their political programmes on the portal and thus opt for a certain candidate. The voter selects the desired candidate and the vote is stored on the database. The voter is allowed to vote only once.

In order to secure anonymous voting, it is necessary to eliminate the possibility to connect the vote to the voter. In addition to anonymity, the security issue has to be covered by using secure communication channels for secure interaction between a client and a server. In order to prevent intrusions into the system 
and to preserve the secrecy of voting the cryptographic protocols should be used. When the voting process is complete, the system can start with a vote counting process.

System for e-voting should:

- provide possibility to check the voter's personal data (checking if the voter is on a registered voters list);

- provide possibility to check whether the voter is voting for the first time or he has already voted;

- provide possibility to the voters to be informed regarding candidates' programs in order to vote for the candidate whose programme matches voter's political views and opinion;

- store votes on a database, whereby there should be a mechanism that will disable linking the vote with its voter, so that voting can be anonymous;

- provide feedback to the voter about successful voting;

- perform vote counting.

\section{4: Research Methodology}

\subsection{Research context and goal}

In order to examine the public opinion regarding the current voting process in the Republic of Serbia and to determine to what extent it is possible to introduce the e-voting system three surveys were conducted. The aim of these surveys was to examine the overall opinion regarding the e-government current state and its advantages and disadvantages, as well as the potential introduction e-voting system in the elections sphere.

The political advantage of the voting process automation for the Republic of Serbia would mean a higher participation and better statistics during the voting process, and a less likely error to occur during vote counting. In the economic sense, the implementation of an e-voting system would lead to voting cost reduction, such as transportation of ballot boxes, procurement of colorless spray and accompanying elements, but also the new working positions would be opened for ICT experts. An e-voting system implementation would attract the attention of ICT experts in order to improve it. In addition, such a system can gain the trust of the voters that their vote can really affect the voting outcome, so it can be an advantage in a social sense. From the technical point of view, new technologies can lead to the encouragement of innovative ideas.

\subsection{Data sample}

Participants in the above mentioned three surveys are presented in Table 1. Questions in these surveys were asked in a manner of determination of the distribution of e-government services as well as familiarity of the the citizens with it.

Table 1: Survey participants

\begin{tabular}{|l|c|}
\hline Participants & Number \\
\hline Citizens & 90 \\
\hline Legal entities & 22 \\
\hline E-government employees & 40 \\
\hline
\end{tabular}

As demographic questions, age and education were taken into consideration in order to get a clearer picture of the potential future users of the e-voting system.

About $90 \%$ of individuals live in Belgrade, $57.8 \%$ belong to the ages between 26 and $30,85.6 \%$ are faculty educated. From the group of respondents of legal entities $27.3 \%$ belong to the ages between 26 and 30 , $90.9 \%$ are faculty educated. From the group of e-government employees the highest percentage of the respondents are between 51 and 60 of age (27.5\%), 62.5\% live in Belgrade and $65 \%$ are faculty educated.

\subsection{Instruments}

There were three surveys - one for each group of respondents. For the private individuals the first six questions referred to defining age, education, city, gender, level of Internet activity and a way of accessing the Internet. The block of nineteen questions related to e-government, in order to examine whether 
participants are familiar with its concept, but also to examine which services individuals considered efficient, which services they have used and what could be advantages and/or disadvantages of e-government. The last twenty-six questions referred to the e-voting. In addition to examining the advantages and/or disadvantages, some of the questions had the goal to investigate whether the respondents were willing to use the e-voting system instead of the traditional voting system, or they found the current voting system reliable.

For the group of legal entities there were also three blocks of questions: general information (six questions), e-government (nineteen questions) and e-voting (twenty-six questions). The questions for legal entities were slightly different from those for individuals since there were questions among them regarding using egovernment services that could represent a benefit for the company.

Finally, the survey for the e-government employees contained seven general questions, nineteen questions regarding e-government and their attitude as employees, and the same twenty-six question as for individuals and legal entities. Questions for this group were in accordance to the respondents' experience before and after the introduction the e-government in order to compare the citizens' trust in the efficiency and quality of e-government operations.

Respondents from all three groups could leave their comments regarding e-government and/or e-voting and suggestions for their improvement.

\subsection{Research results}

The highest percentage of the individual respondents used e-government services as private individuals (53.9\%). Among the most commonly used services respondents cited Personal documents (68.8\%), Registry books (45.3\%) and Employment search (15.6\%).

E-government services which were stated by the group of legal entities as the most commonly used by legal entities were: Value added tax (47.1\%), Statistical reporting (47.1\%), Contributions for employees $(35.3 \%)$ and Public procurement (29.4\%). Among the respondents in this group $63.2 \%$ consider that application of e-government services represents an advantage for the company and improves its business.

Among the respondents from the group of e-government employees $64.1 \%$ consider that the process of introduction and implementation of e-government represents an advantage and a positive effect on their work. As employees in Government, 33.3\% consider that citizens have confidence in proper functioning and operation of e-government, while $65 \%$ consider that the process of ordering personal documents, as well as scheduling the appointment is facilitated this way. On the other hand, as users of e-government services, respondents state Registry books (74.3\%), Personal documents (68.6\%), Cars registration $(22.9 \%)$ and Public procurement $(22.9 \%)$ as the most commonly used services.

\subsubsection{Comparative analysis}

It is important to emphasize that most of the respondents had suggestions for the improvement of the voting process in the Republic of Serbia which refer to introducing the e-voting system. Some of the respondents suggested recruiting more qualified IT staff which will help and support citizens to use the e-voting system.

Half of the respondents that belong to the group of private individuals consider that the introduction of the concept of e-voting on the territory of the Republic of Serbia could increase the interest of the voters to participate in the election process. For legal entities this percentage is $40.9 \%$, i.e., $53.9 \%$ for e-government employees. It can be concluded that, although the samples for these three groups are different, respondents from all these groups have mainly provided similar answers to the presented statements.

Based on the answers, it can be concluded that respondents recognize the real issues regarding the evoting introduction process. When it comes to transparency, respondents from all three groups find that the e-voting system can mean information availability; thus $67.9 \%$ respondents from the group of private individuals agree with this statement, as well as $70 \%$ from the group of legal entities and $71.8 \%$ from the group of e-government employees. 
Table 2 presents the comparative analysis of negative, neutral and positive attitudes of the citizens, egovernment employees and legal entities to the questions divided into four categories: cost reduction, ease of use, efficiency improvement and IT knowledge \& security issues. These categories are considered as the most critical points when it comes to the e-voting system introduction process. Thus, it is necessary to investigate into which of these categories require improvement.

For the purposes of detailed analysis questions for each category are divided into two more groups questions regarding e-government and questions regarding e-voting. In this way it is possible to clearly establish the negative aspects from the respondents' standpoint that should be emphasized in order to promote and improve e-participation.

Table 2: Comparative review of negative, neutral and positive attitudes by question categories

\begin{tabular}{|c|c|c|c|c|c|}
\hline & & & Negative & Neutral & Positive \\
\hline \multirow{6}{*}{ Cost reduction } & \multirow{3}{*}{ E-government } & Citizens & $4.12 \%$ & $13.42 \%$ & $82.46 \%$ \\
\hline & & Employees & $8.75 \%$ & $20.00 \%$ & $71.25 \%$ \\
\hline & & Legal entities & $5.00 \%$ & $5.00 \%$ & $90.00 \%$ \\
\hline & \multirow{3}{*}{ E-voting } & Citizens & $4.06 \%$ & $11.58 \%$ & $84.36 \%$ \\
\hline & & Employees & $11.88 \%$ & $21.00 \%$ & $67.12 \%$ \\
\hline & & Legal entities & $0.00 \%$ & $15.00 \%$ & $85.00 \%$ \\
\hline \multirow{6}{*}{ Ease of use } & \multirow{3}{*}{ E-government } & Citizens & $5.23 \%$ & $25.02 \%$ & $69.74 \%$ \\
\hline & & Employees & $8.50 \%$ & $27.00 \%$ & $64.50 \%$ \\
\hline & & Legal entities & $9.02 \%$ & $22.68 \%$ & $68.31 \%$ \\
\hline & \multirow{3}{*}{ E-voting } & Citizens & $5.95 \%$ & $15.75 \%$ & $78.31 \%$ \\
\hline & & Employees & $11.98 \%$ & $17.48 \%$ & $70.54 \%$ \\
\hline & & Legal entities & $7.86 \%$ & $14.08 \%$ & $78.06 \%$ \\
\hline \multirow{6}{*}{$\begin{array}{c}\text { Efficiency } \\
\text { improvement }\end{array}$} & \multirow{3}{*}{ E-government } & Citizens & $3.57 \%$ & $25.84 \%$ & $70.59 \%$ \\
\hline & & Employees & $13.45 \%$ & $19.32 \%$ & $67.23 \%$ \\
\hline & & Legal entities & $7.23 \%$ & $15.78 \%$ & $76.99 \%$ \\
\hline & \multirow{3}{*}{ E-voting } & Citizens & $12.25 \%$ & $25.88 \%$ & $61.87 \%$ \\
\hline & & Employees & $17.80 \%$ & $24.76 \%$ & $57.44 \%$ \\
\hline & & Legal entities & $6.08 \%$ & $31.33 \%$ & $62.59 \%$ \\
\hline \multirow{6}{*}{$\begin{array}{l}\text { IT knowledge \& } \\
\text { security issue }\end{array}$} & \multirow{3}{*}{ E-government } & Citizens & $54.03 \%$ & $32.56 \%$ & $13.41 \%$ \\
\hline & & Employees & $46.04 \%$ & $34.59 \%$ & $19.37 \%$ \\
\hline & & Legal entities & $59.72 \%$ & $20.69 \%$ & $19.58 \%$ \\
\hline & \multirow{3}{*}{ E-voting } & Citizens & $54.36 \%$ & $23.28 \%$ & $22.36 \%$ \\
\hline & & Employees & $59.02 \%$ & $20.34 \%$ & $20.64 \%$ \\
\hline & & Legal entities & $54.64 \%$ & $24.93 \%$ & $20.43 \%$ \\
\hline
\end{tabular}

In table 3 the four categories are presented along with the explanation to which types of questions they refer.

Observing the e-voting process as an asset for cost reduction, $85 \%$ of legal entities consider that such a process can involve not only cost savings of administrative functions, but also the voting process itself in terms of organizing the voting stations with ballot boxes and ballot papers. The positive response about the cost reduction was obtained from $84.36 \%$ of the citizens and $67.12 \%$ of the e-government employees. Figure 4 shows the ratio of negative, neutral and positive responses of citizens, e-government employees and legal entities to the questions from the cost reduction category. 
Table 3: Review of question types by categories

\begin{tabular}{|c|c|}
\hline Cost reduction & $\begin{array}{l}\text { - Reducing cost of the overall voting process } \\
\text { - } \quad \text { Reducing cost of administrative functions } \\
\text { - Time management }\end{array}$ \\
\hline Ease of use & $\begin{array}{l}\text { - } \quad \text { Ease of use and access } \\
\text { - } \quad \text { Information transparency and availability } \\
\text { - } \quad \text { Remote access } \\
\text { - } \quad \text { Usefulness and applicability } \\
\text { - Increasing the voters' interest in participation }\end{array}$ \\
\hline Efficiency improvement & $\begin{array}{l}\text { - } \text { Accuracy in paper management } \\
\text { - } \text { Accuracy of services } \\
\text { - } \text { Faster and simpler service } \\
\text { - } \text { Efficiency enhancement } \\
\text { - } \text { Impartion of the human factor } \\
\text { Imp the voting results }\end{array}$ \\
\hline $\begin{array}{c}\text { IT knowledge \& security } \\
\text { issue }\end{array}$ & $\begin{array}{ll}\text { - } & \text { Identification issues } \\
\text { - } & \text { Data privacy issues } \\
\text { - } & \text { Lack of qualified IT staff } \\
\text { - } & \text { Lack of IT knowledge } \\
\text { - } & \text { Security issues } \\
\text { - } & \text { Limited Internet access } \\
\quad \text { Malicious programs (viruses) }\end{array}$ \\
\hline
\end{tabular}

From the aspect of the ease of use, $78.31 \%$ respondents from the group of individuals, $70.54 \%$ e-government employees and $78.06 \%$ legal entities consider that such a system will be easy to use. Graphical representation of negative, neutral and positive responses to this category is shown in Figure 5.

$81.2 \%$ individuals find this system useful and applicable for public use, while $83.3 \%$ respondents would gladly use this kind of e-voting system. These results show that it is possible to do additional statistical analysis which can be used as basis for determining the level of the real usefulness and ease of use. $81.8 \%$ respondents from the group of legal entities agree that this ccan be a user-friendly system; $90.9 \%$ consider that this kind of e-voting system can be useful and applicable for public use; and $81.8 \%$ would gladly use this system. From the aspect of simplicity of use, 55\% e-government employees agree with the statement that the e-voting system can be user-friendly; $67.5 \%$ that it can be useful and applicable, while $77.5 \%$ would gladly use this kind of e-voting system.

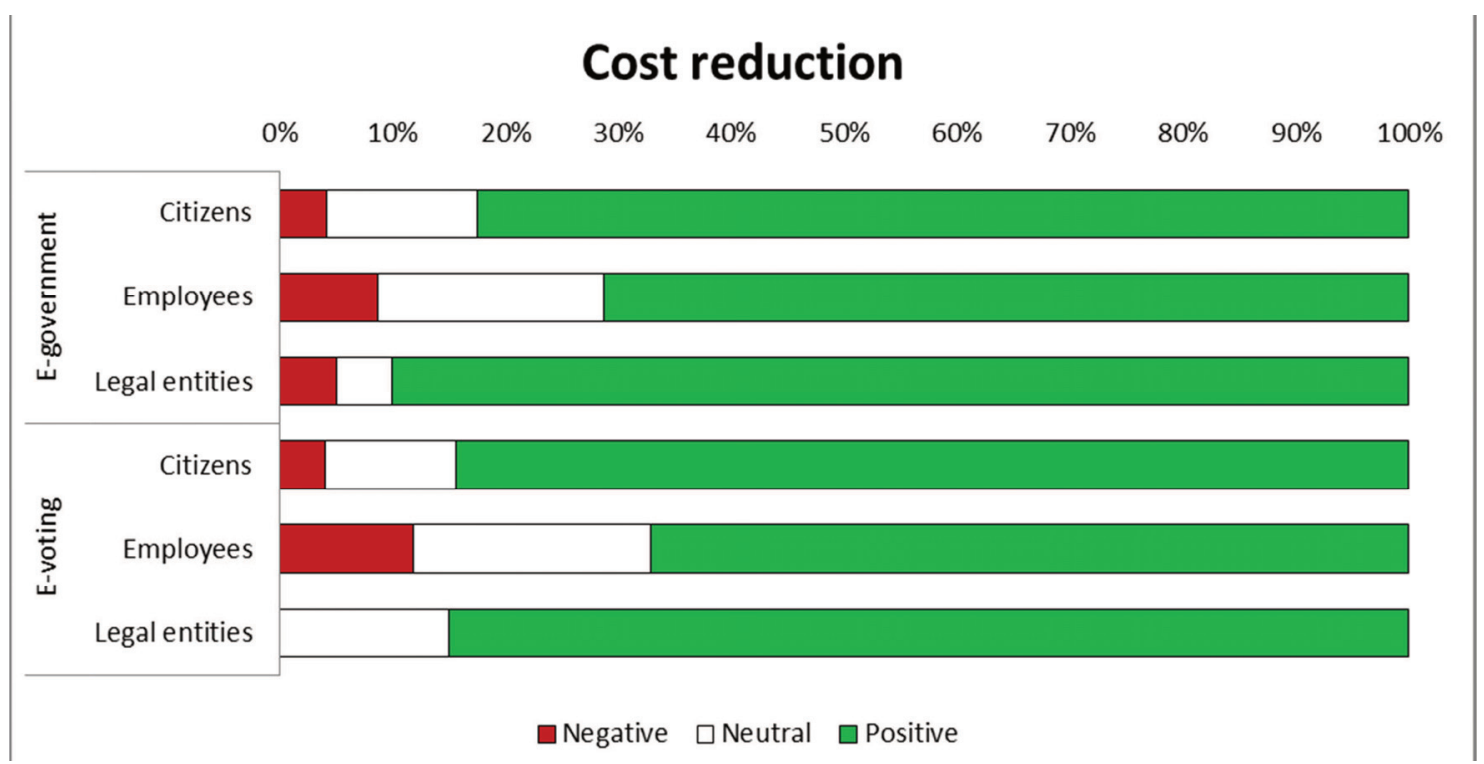

Figure 4: Graphical view of the negative, neutral and positive answers regarding cost reduction 


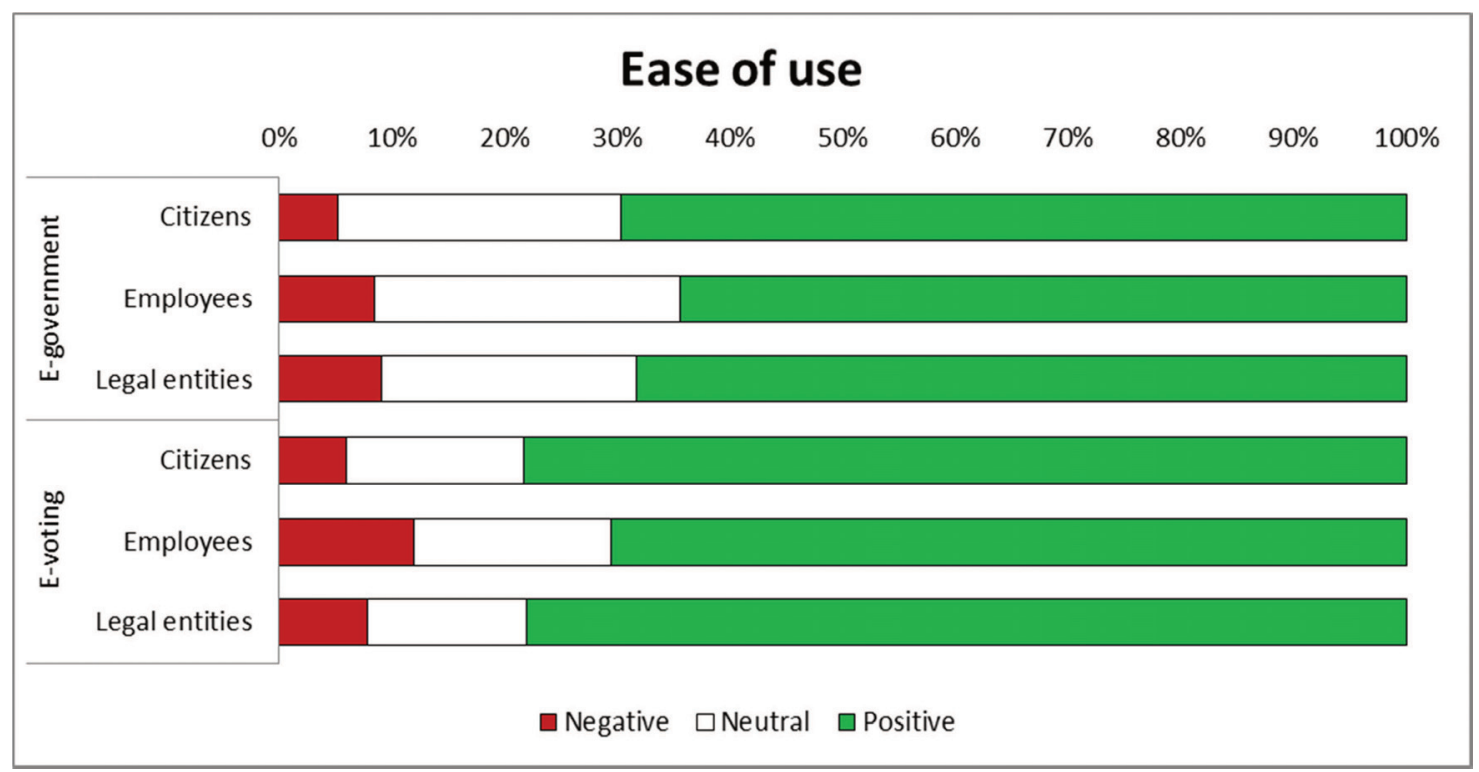

Figure 5: Graphical view of the negative, neutral and positive answers regarding ease of use

Response to the questions regarding efficiency improvement by implementing and using the e-voting system was positive, $61.87 \%$, from individuals and $57.44 \%$ from e-government employees and $62.59 \%$ from legal entities. Graphical view of the ratio of negative, neutral and positive answers of the all three groups of respondents to the questions from the efficiency improvement category is presented in Figure 6.

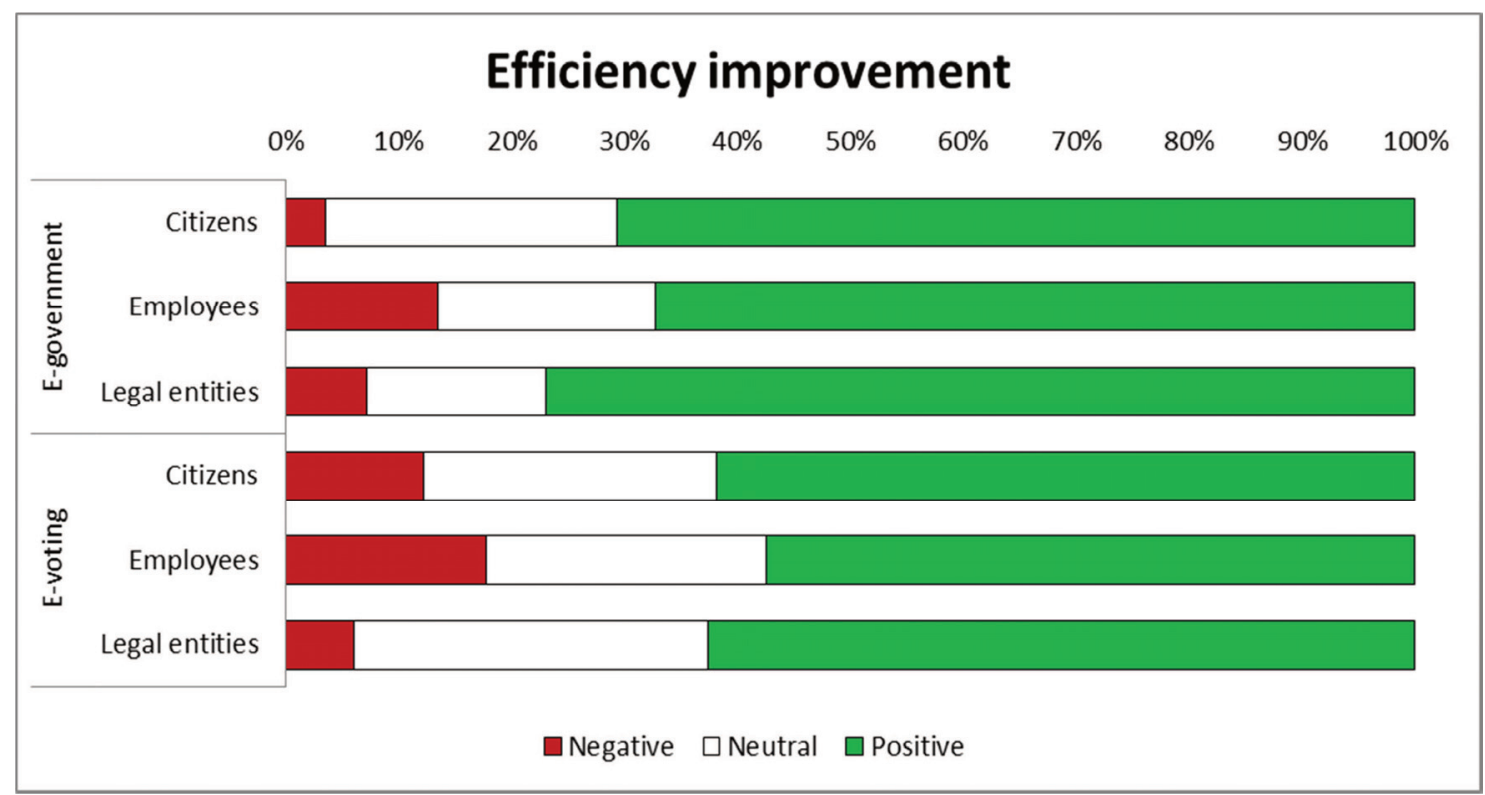

Figure 6: Graphical view of the negative, neutral and positive answers regarding efficiency improvements

As shown in Table 1 and in Figure 7, the most critical issue of the e-voting introduction process from the standpoint of the e-government employees is IT knowledge and security issue; $59.02 \%$ of the respondents from this group have concerns regarding the lack of IT knowledge as this may have a negative impact to the e-voting process. This statement is supported by $54.36 \%$ individuals and $54.64 \%$ legal entities. 


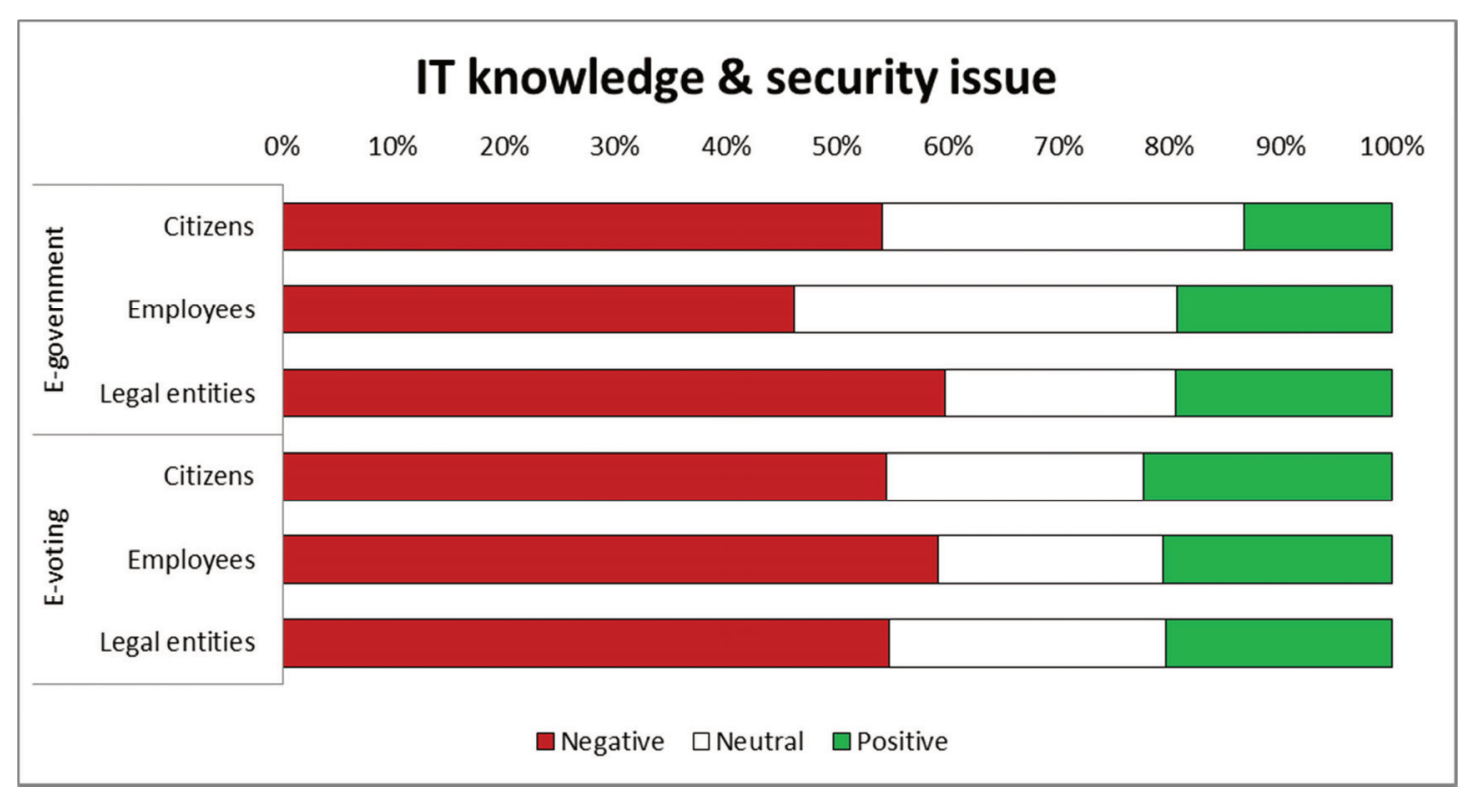

Figure 7: Graphical view of the negative, neutral and positive answers regarding

IT knowledge and security issue

Security issue is also considered as a critical point that should be overcome so as not to endanger the normal flow of the voting process. By using the e-voting system from personal computer the crucial issue could be malicious programs (viruses) that can be located on the client's computer. This could affect the accuracy of voting in that it may change the vote. This statement is agreed upon by $64.6 \%$ of individuals, $60 \%$ of legal entities and $69.2 \%$ of e-government employees.

The identity issue in e-voting process is seen as critical by $73.2 \%$ individuals and $73.6 \%$ e-government employees, but only $57.9 \%$ respondents from the group of legal entities agree with this statement. The mistrust of the citizens concerning new technologies is recognised by $85 \%$ of respondents of legal entities as a real issue in e-voting introduction process, as well as by $77.1 \%$ of respondents from individual's group and $76.3 \%$ from e-government employees. The limited Internet access in many municipalities in the Republic of Serbia can prove a serious obstacle to introduction and implementation of the e-voting process. $71.2 \%$ individuals, $75 \%$ legal entities and $52.7 \%$ e-government employees agreed with this statement.

The overall opinion based on responses of all three groups is that the crucial issue is security, but with the use of new information technology and with the support of qualified staff, the e-voting process can provide more advantages than the traditional "paper" voting.

\section{Conclusion and Discussion}

In this paper the public opinion was analyzed regarding introducing the e-voting system for the potential purposes of the election process in the Republic of Serbia, and the solution for development and implementation of e-voting system was proposed. The main objective was to examine whether there was a possibility to introduce the e-voting system.

Surveys show that citizens, e-government employees and legal entities generally agree with the concept of e-voting system and the idea of introducing the online system that could improve the election process regarding reducing errors and human factor ( $\mathrm{H} 1$ was confirmed), but also to provide accessibility to persons with special needs.

The development and implementation of the e-government services model in the e-voting area can improve the quality and process of providing government services; simplification of the voting process increases the satisfaction of citizens, the accuracy of results and spreads awareness about further development of electronic government services. The development of such an e-voting model represents the progress of the information society in a way of increasing the use of e-government services to a higher level. The voting process automation can imply a broader participation of citizens in the election process due to an open Internet access and the application of IC technologies. In this way, social changes are promoted, mutual learning is ensured and successful solutions are spreading, but also the room for partnership between government and non-government organization is created which leads toward meeting long-term technological requirements of the ICT. The aim of the e-voting system is to contribute to the efficiency increment and cost reduction during elections, whether they 
are parliamentary or other elections. The system will allow the voters to be more informed regarding programmes of candidates, in order to make the best decision with all necessary information and in a best way. Research of the issues of the application the e-voting system from a social utility point of view can have several implications: research results can help in the analysis of further implementation and integration of e-voting system in wider use as one of e-government services; research results can contribute to precisely determine time, material and human resources, as well as legal regulations for introducing the e-voting system; research results can help identify the opportunities and project boundaries of the development of e-voting and voting advice systems.

The possibility of applying research results are reflected in the following:

- the e-voting system can be used not only for parliamentary, presidential or local elections, but for elections beyond political framework;

- the application of the e-voting system can increase the number of voters interested in participation in voting, due to the knowledge that votes are counted automatically, i.e., independently of human factor, and thus less likely to be lost;

- by using the voting advice system it is possible to vote for the candidate whose programme meets the political views and attitude of a voter, thus with a voting support the voter can make the best decision.

As e-voting systems are applied worldwide, which significantly reduces the possibility of errors to occur, it is appropriate to apply the research results on the voting system of the Republic of Serbia.

The results can be used as a starting point for the future research. Based on this analysis it is possible to develop an evoting system with the active participation of citizens as well as legal entities, and with the support of e-government employees. This will cover all the aspects of good and transparent e-government with required experience and good cooperation between the private and the public sectors.

In this paper we propose an integration model of e-voting services, IT infrastructure and participants register. By implementing and integrating these components in a mobile or web application we can get an e-voting system with additional module for counting the votes and reporting the results. The most important criterion that should be met is security, so it is necessary to use the cryptographic protocols in order to increase security to a higher level. This e-voting system should be reliable, so that voters can be sure that their votes will not be changed; and scalable, so that a large number of users (voters) can access the application at the same time without reducing the response speed.

There are some recommendations and implications both for academia and practitioners.

For academia:

- It is necessary to seek for new solutions that solve the security issues and prevent malicious intrusions to the network.

- The new technologies and their application in the e-voting service are of a great importance.

- As long as there are security systems, there will also be malicious intrusions. Therefore, the security issue should never be ignored. In fact, it is necessary to strive towards new technologies that provide efficiency, increase the speed of data processing and data accuracy.

For practitioners:

- In order to gain citizens' trust regarding the work of the e-voting system and its efficiency, it is important to include them into the implementation process.

- In this way it is possible to develop a reliable system which will not be difficult for the citizens to use.

- It is certainly necessary to provide a clear and precise instructions for using the system.

- The emphasis should be on the ease of use, remote access, reliability and security of the e-voting system.

In addition, the model could be used in different contexts, such as education, edutainment, etc., however, with small adjustments.

As regerds the future development, the area which will be researched refers to the implementation of mobile technologies in the e-voting model. As the social networks are in the focus of current developments, the possibility to integrate the application with social networks should be considered. Also, inclusion the media could have a strong influence on the process of introducing the e-voting system in public use, since the media function is a social responsibility and as such it acts in the public and individual interest.

\section{REFERENCES}

[1] Al-Hakim, L. (2007). Global E-government: Theory, Applications and Benchmarking. Toowoomba: Idea Group Inc (IGI). doi:10.4018/978-1-59904-027-1

[2] Alkassar, A., \& Volkamer, M. (2007). E-Voting and Identity. First International Conference, VOTE-ID 2007. Bochum, Germany: Springer Science \& Business Media, 2007. DOI:10.1007/978-3-540-77493-8

[3] Anderson, D. R. (2006). The critical importance of sustainability risk management. Journal of Risk and Insurance, Vol. 53, no. 4. 
[4] Anthopoulos, L., Siozos, P., \& Tsoukalas, I. (2007). Applying participatory design and collaboration in digital public services for discovering and re-designing e-Government services. Government Information Quarterly 24, 353-376. doi:10.1016/j.giq.2006.07.018

[5] Brown, M. (2003). Electronic government. U J. Rabin, Encyclopedia of Public Administration and Public Policy: A-J (str. 427-432). CRC Press.

[6] Drechsler, W. (2004). The Estonian e-Voting Laws Discourse: Paradigmatic Benchmarking for Central and Eastern Europe. NISPAcee Occasional Papers in Public Administration and Public Policy, Volume V - No. 2.

[7] Fossen, T., \& Anderson, J. (2014). What's the point of voting advice applications? Competing perspectives on democracy and citizenship. Electoral Studies, 36, 244-251. doi:10.1016/j.electstud.2014.04.001

[8] Frias-Aceituno, J., Garcia-Sanchez, I., \& Rodriguez-Dominguez, L. (2014). Electronic Administration Styles And Their Determinants. Evidence From Spanish Local Governments. Transylvanian Review of Administrative Sciences, 90-108.

[9] Hite, R. C. (2004). Elections electronic voting offers opportunities and presents challenges. DIANE Publishing.

[10] IDEA, I. (2011). Introducing Electronic Voting: Essential Considerations. Preuzeto Jun 2015 sa International Institute for Democracy and Electoral Assistance: http://www.eods.eu/library/IDEA.IntroducingElectronic-Voting-Essential-Considerations.pdf

[11] IRMA, I. R. (2015). Public Affairs and Administration: Concepts, Methodologies, Tools, and Applications: Concepts, Methodologies, Tools, and Applications. IGI Global, 2015.

[12] Ke, W., \& Wei, K. K. (2004). Successful E-Government in Singapore. Communications of the ACM 47(6), 95-99. DOI:10.1145/990680.990687

[13] Kreca, M., \& Vaskovic, J. (2013). Analiza stanja servisa elektronske uprave u Srbiji. Infotech. Aranđelovac.

[14] Ladner, A., \& Pianzola, J. (2010). Do Voting Advice Applications Have an Effect on Electoral Participation and Voter Turnout? Evidence from the 2007 Swiss Federal Elections. Lecture Notes in Computer Science Volume 6229, 211-224. DOI:10.1007/978-3-642-15158-3_18

[15] Ladner, A., \& Pianzola, J. (2014). Voting Advice Applications. U M. Khosrow-Pour, Information Science and Technology. DOI:10.1016/j.electstud.2014.06.010

[16] Lee, J. (2010). 10 year retrospect on stage models of e-Government: A qualitative meta-synthesis. Government Information Quarterly 27, 220-230. doi:10.1016/j.giq.2009.12.009

[17] Maletic, M., Barac, D., Naumovic, T., Bogdanovic, Z., \& Radenkovic, B. (2018). Blending crowdvoting in modern e-learning environments. The International Review of Research in Open and Distributed Learning.

[18] Malidzan, V. (2011). Kriptološki protokoli u e-glasanju. INFOTEH (str. 621-625). Jahorina: INFOTEH-Jahorina Vol. 10, Ref. E-III-8.

[19] Markovic, N., Stoimenov, L., Vojinović, O., \& Milentijević, I. (2008). E-Uprava u Srbiji: Pregled postojećeg stanja javnih servisa. Beograd: 16. Telekomunikacioni forum Telfor.

[20] Mauw, S., Verschuren, J., \& de Vink, E. (2007). Data Anonymity in the FOO Voting Scheme. Electronic Notes in Theoretical Computer Science 168, 5-28. doi:10.1016/j.entcs.2006.11.001

[21] Nica, E., Popescu, G., Nicolaescu, E., \& Constantin, V. (2014). The Effectiveness of Social Media Implementation at Local Government Levels. Transylvanian Review of Administrative Sciences, 152-166.

[22] OECD. (2003). The e-Government Imperative. Paris: OECD Publications. doi:10.1787/19901054

[23] Park, H. M. (2007). How Does Information and Communication Technology Affect Civic Engagement? An Analysis Focusing on Electronic Government and Campaign Websites. ProQuest.

[24] Ramonaite, A. (2010). Voting Advice Applications in Lithuania: Promoting Programmatic Competition or Breeding Populism? Policy \& Internet, Volume 2, Issue 1, 117-147. doi:10.2202/1944-2866.1027

[25] Reddick, C. G., \& Frank, H. A. (2007). The perceived impacts of E-Government on U.S. cities: A survey of Florida and Texas city managers. Government Information Quarterly, 24(3), 576-594. doi:10.1016/j.giq.2006.09.004

[26] Rennie, E. (2008). Growing Green, Boosting the bottom line with sustainable business practices . APICS Magazine. Vol. 18, no. 2.

[27] Sakowicz, M. (2004). How Should e-Government Be Evaluated? Different Methodologies and Methods. NISPAcee Occasional Papers in Public Administration and Public Policy, Volume V - No. 2.

[28] Sandoval-Almazan, R., \& Gil-Garcia, J. (2012). Are government internet portals evolving towards more interaction, participation, and collaboration? Revisiting the rhetoric of e-government among municipalities. Government Information Quarterly 29, S72-S81. doi:10.1016/j.giq.2011.09.004

[29] Soliman, K., \& Affisco, J. (2006). E-government. Emerald Group Publishing. doi:10.1108/14637150610643724 
[30] Spacek, D. (2014). Coordination of and Through E-Government: The Case of the Czech Republic. NISPAcee Journal of Public Administration and Policy. Volume 7, Issue 1, 83-106. doi:10.2478/nispa2014-0001

[31] Veljkovic, N., Bogdanovic-Dinic, S., \& Stoimenov, L. (2011). Od E-uprave 1.0 do otvorene uprave: Aktuelne inicijative za dalji razoj E-uprave. Kopaonik: Informaciono društvo Srbije: Yu Info.

[32] Vote Match Europe. (2014). Preuzeto sa http://www.votematch.eu/

[33] Vote Match UK. (2014). Preuzeto sa http://www.votematch.org.uk/

[34] Vrhovsek, M., \& Spalevic, Z. (2011). The Perspectives of Development of Electronic Government in Serbia and EU Experience. Megatrend Review.

Received: 2018-10-17

Revisions requested: 2019-01-14

Revised: 2019-01-30

Accepted: 2019-02-21

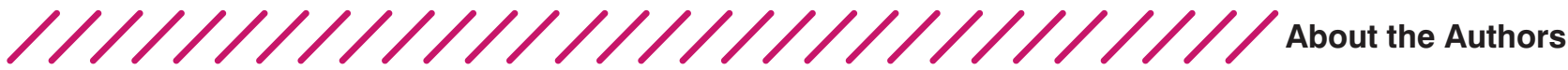

Marija Maletić

DXC.Technology - Enterprise Services, Belgrade, Serbia e-mail: maletic.mari@gmail.com

PhD student at the Department of E-business at the Faculty of Organizational Sciences, University of Belgrade. She works for the DXC.Technology - Enterprise Services company as Release and Testing Specialist. Her research interests are: E-banking, E-government, E-education, Internet Technologies, Mobile Technologies, Operational Research and Statistics.

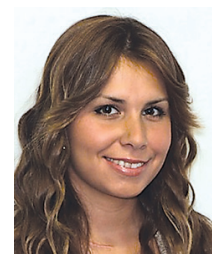

Dušan Barać

University of Belgrade, Faculty of Organizational Sciences, Serbia e-mail: dusan.barac@fon.bg.ac.rs

Associate professor at the Department of E-business at the Faculty of Organizational Sciences, University of Belgrade. His research interests are: E-business, E-commerce, Web technologies and mobile business.

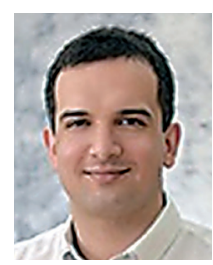

Vuk Rakočević Embassy of Montenegro in the Republic of Serbia, Serbia e-mail: vuk.rakocevic@mfa.gov.me

Consul at the Embassy of Montenegro in the Republic of Serbia, Belgrade. His research interests are: E-government, E-diplomacy, Business law.

Tamara Naumović University of Belgrade, Faculty of Organizational Sciences, Serbia e-mail: tamara.naumovic@fon.bg.ac.rs

PhD student and a teaching associate at the Department of E-business at the Faculty of Organizational Sciences, University of Belgrade. Her research interests are: E-business, Internet Technologies, Mobile Technologies, Computer Simulation.

Artur Bjelica

University of Novi Sad, Faculty of Medicine, Serbia e-mail: arturbjelica@gmail.com

Associate professor at the Faculty of Medicine, University of Novi Sad. His research interests are: E-health, Gynecology, Pregnancy care, Pervasive computing. 